\title{
Short-term wind speed prediction based on MLP and NARX network models
}

\author{
Yousra Amellas ${ }^{1}$, Outman El Bakkali ${ }^{2}$, Abdelouahed Djebil ${ }^{3}$, Adil Echchelh ${ }^{4}$ \\ ${ }^{1,4}$ Laboratory of Materials and Energy, Faculty of Science, University of Kenitra, Morocco \\ ${ }^{1,3}$ Laboratory of Energetics, Fluid Mechanics and Materials, Faculty of Science, University of Tetouan, Morocco \\ ${ }^{2}$ Laboratory of Semi-Conductors and Thin Layers, Faculty of Science, University of Kenitra, Morocco
}

\begin{tabular}{l} 
Article Info \\
\hline Article history: \\
Received Jun 24, 2019 \\
Revised Sep 25, 2019 \\
Accepted Oct 16, 2019 \\
\hline
\end{tabular}

Keywords:

Artificial neural network

Daily prediction

Multi-layer perceptron (MLP)

NARX

Recurrent neural network (RNN)

\begin{abstract}
The article aims to predict the wind speed by two artificial neural network's models. The first model is a multilayer perceptron (MLP) treated by backpropagation algorithm and the second one is a recurrent neuron network type, processed by the NARX model. The two models having the same Network's structure, which they are composed by 4 Inputs layers (Wind Speed, Pressure Temperature and Humidity), an intermediate layer defined by 20 neurons and an activation function, as well as a single output layer characterized by wind speed and a linear function. NARX shows the best results with a regression coefficient $\mathrm{R}=0.984$ et $\mathrm{RMSE}=0.314$.
\end{abstract}

Copyright $@ 2020$ Institute of Advanced Engineering and Science. All rights reserved.

\section{Corresponding Author:}

Yousra Amellas,

Laboratory of Materials and Energy,

Faculty of Science, University of Kenitra, Morocco.

Email: yousra.ame@gmail.com

\section{INTRODUCTION}

The forecast of the wind speed is an essential process in the management of electricity generation systems of wind origin, it allows the provision of the necessary information to elaborate good strategies and to achieve the planned objectives to optimize efficiency, decreasing and stabilizing the difference between demand and offer [1]. Several meteorological, mechanical and electrical characteristics allowd an optimal electric energy. The Wind is the key element that is usually characterized by his speed and his direction which have the strong influence on the final production, other variables are taken into consideration as the pressure, temperature, humidity and the dew point temperature, they are all unpredictable and random. There are several techniques for predicting and anticipating the future. Two broad categories of approaches have been developed, Physical approaches such as NWP (Numeric Weather Predictors), GFS (Global forecast system), HIRLAM [2] are usually applied for the long-term forecast. While for the short-term forecast statistical approaches have shown effective and efficient results. They integrate two broad methods time series like ARMA, ARIMA, Grey Predictors, Linear Predictions, ARX... usually considered as linear methods that they deal linear and simple models. For non-linear and complex models, the second method which is artificial intelligence (AI) [3] presents good results include, artificial neural networks (ANN), Support Vector Machine (SVM), Fuzzy Logic Methods, Wavelet Predictions [4, 5] ...as well as other hybrid models as ANFIS. This study deals with the 24-hour wind forecast taking as an example 3 years of meteorological data (2015-2016-2017) of the Tetouan region. We chose supervised learning for our regression problem using two different types of neural networks. The multilayer perceptron (MLP) type of feed-forward neural network (FNN) based on back-propagation algorithm using Levenberg-Marquardt training function (LM). The second is Nonlinear Autoregressive exogenous model (NARX) type of Recurrent 
neural network (RNN) based on Time series using Scaled Conjugate Gradient (SGC) training function [6]. We found that the latter leads to better results. To evaluate the performance of the model and select a better structure. We have based on MSE and RMSE estimators.

\section{RESEARCH METHOD}

\subsection{Meteorological Data}

Through this study we want to choose the most performing predictive model of the daily wind speed of Tetouan site. Meteorological data of the Tetouan site have been taken every day for three years, 20152016-2017; they are obtained from the site https://www.historique-meteo.net/afrique/maroc/tetouan/. The Table 1 meteorological datasheet includes the date (TIME) day by day, Wind speed (WS) in $\mathrm{m} / \mathrm{s}$, Temperature (TMP) in ${ }^{\circ} \mathrm{C}$, Atmospheric pressure (ATM) in $\mathrm{hPa}$, Dew point temperature (DEW) in ${ }^{\circ} \mathrm{C}$ and Humidity $(\mathrm{H})$ in \%. Also the table preprocesses meteorological data to facilitate their use and exploitation [7].

Our meteorological data was processed by Excel software as well as the modeling was done by NNTOOL for MLP and NNSTART for NARX on MATLAB software. Moreover, meteorological data pretreatment simplifies the choice of the parameters of the predictive models. To avoid expensive calculation, it's preferable to minimize the number of model's input variables.

Table 1. Presentation of the Weather Data Sheet of Tetouan

\begin{tabular}{cccccc}
\hline Date & Wind Speed $(\mathrm{m} / \mathrm{s})$ & Pressure $(\mathrm{hPa})$ & Temperature $\left({ }^{\circ} \mathrm{C}\right)$ & Humidity $(\%)$ & Dew point temperature $\left({ }^{\circ} \mathrm{C}\right)$ \\
\hline $30 / 12 / 2017$ & 1,666666667 & 1028 & 15 & 74 & 13 \\
$29 / 12 / 2017$ & 3,611111111 & 1024 & 15 & 87 & 13 \\
$28 / 12 / 2017$ & 6,666666667 & 1024 & 13 & 86 & 12 \\
$27 / 12 / 2017$ & 10 & 1027 & 10 & 87 & 12 \\
$26 / 12 / 2017$ & 6,944444444 & 1028 & 12 & 83 & 10 \\
$25 / 12 / 2017$ & 1,944444444 & 1031 & 12 & 66 & 11 \\
$24 / 12 / 2017$ & 3,611111111 & 1032 & 12 & 63 & 7 \\
$23 / 12 / 2017$ & 2,777777778 & 1037 & 14 & 69 & 7 \\
$22 / 12 / 2017$ & 2,777777778 & 1032 & 11 & 69 & 8 \\
$21 / 12 / 2017$ & 5,555555556 & 1029 & 11 & 75 & \\
$20 / 12 / 2017$ & 5,277777778 & & & \\
\hline
\end{tabular}

\subsection{Neural Network}

A neural network is a model which is described usually by many components. Usually characterized by inputs $\left(\mathrm{x}_{0}, \ldots, \mathrm{x}_{\mathrm{j}}\right)$ come from previous neuron, every input is associated to a weight $w$ representing connection strength, their values are calculated in the learning phase in hidden layer, it is constituted of several neurons as well as activation function $\mathrm{a}=\sum\left(\mathrm{w}_{\mathrm{ij}} \cdot \mathrm{x}_{\mathrm{i}}\right)\left(\mathrm{x}_{\mathrm{j}}\right.$ present the inputs of the network, and $\mathrm{w}_{\mathrm{ij}}$ present the weights associated to each neuron) it can be presented in different types, the most used are, linear function, hyperbolic-tangent et sigmoid function, and an output layer generally characterized by a linear function [8-9]. Formal neuron presentation as shown in Figure 1.

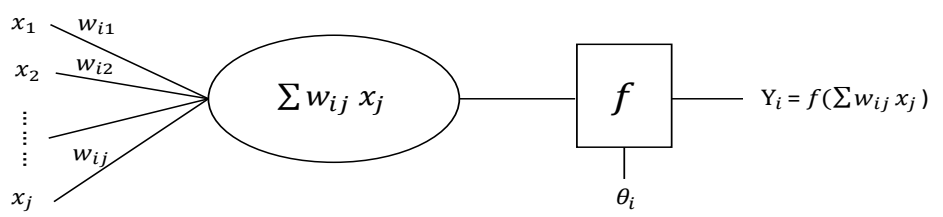

Figure 1. Formal neuron presentation

$x_{j}:$ Input.

$w_{j}$ : Connection weight.

$\sum w_{i j} x_{i}:$ The weighted sum of the Inputs $x_{j}$ weighted by $w_{j}$.

$f:$ Activation function.

$\theta_{i}:$ Threshold.

$Y_{i}:$ Output. 
As previously mentioned we used two types of neural networks. The MLP [10] is a classic neuron network which consists of an input, hidden and an output layers based on the error backpropagation algorithm which is mainly based on the gradient descent algorithm. The backpropagation algorithm measures the error between the desired output $\mathrm{d}$ and outputs $\mathrm{O}$ calculated by the network. To minimize the error, the information is propagated from the output to the input by adjusting the weights [11]. There are other types of classical neural networks that belong to backpropagation family as: Adaline (Adaptive Linear Neuron), Madaline (Multi-Adaline) also RBF (Radial Basis Function) is structurally same as perceptron MLP. Example of multilayer perceptron with a hidden layer and an output layer as shown in Figure 2.

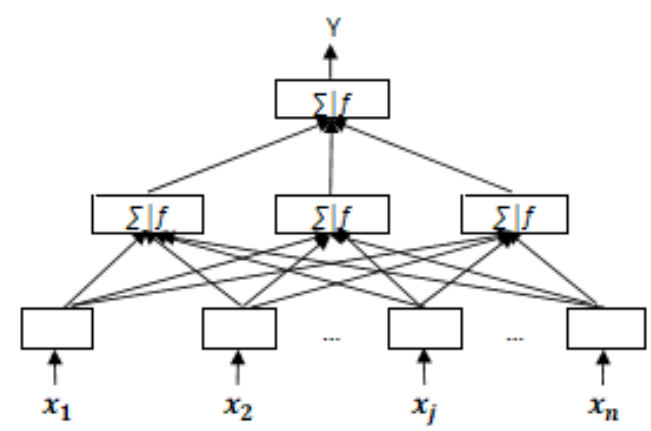

\author{
Output layer \\ Hidden layer \\ Input layer
}

Figure 2. Example of multilayer perceptron with a hidden layer and an output layer

While NARX is a type of recurrent neural network who has the same training techniques that classical networks based on the gradient back-propagation algorithm. To compare the two types of networks we keep the same structure of the network. NARX is characterized by its great power to analyze non-linear time series like dynamic systems as well as the descent gradient algorithm behaves efficiently and converges quickly than in other networks. There are others types of deep RNN called Elman Neural Network [12] LSTM (Long Short-Term Memory) [13] and GRU (Gated Recurrent Unit). Example of NARX-NN structure (close loop and open loop) as shown in Figures 3 and 4.

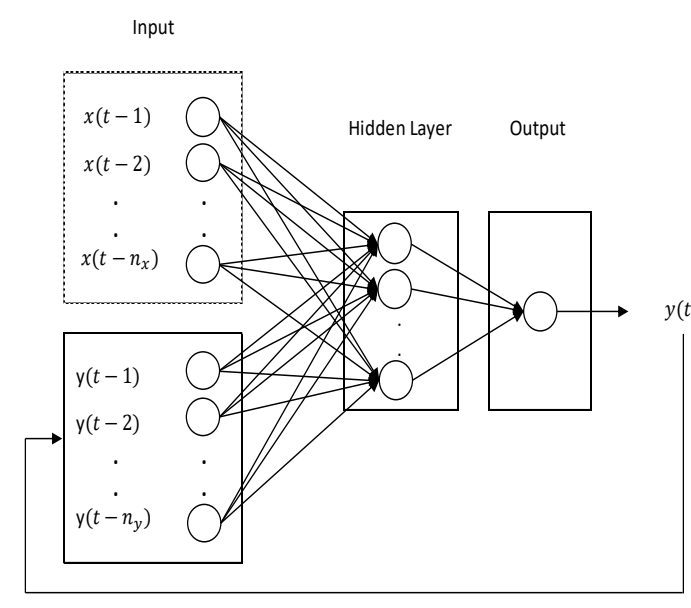

Figure 3. Example of NARX-NN structure (close loop)

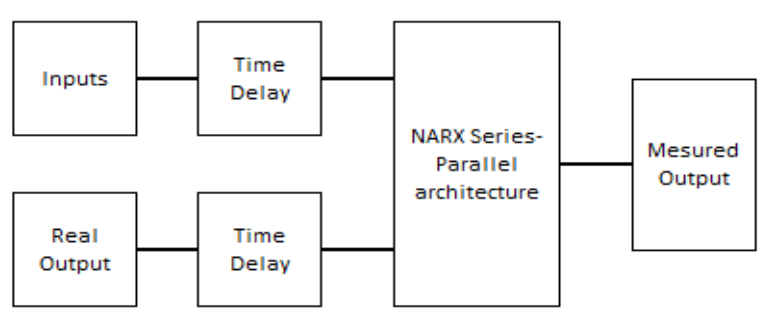

Figure 4. Example of NARX-NN structure (open loop)

Some studies case has been analyzed by NARX for Hourly Solar Radiation Prediction. Of course, there are others case studies who have used NARX for forecasting wind speed.

Erasmo \&Wilfrido [14] did the study on two sites in Mexico comparing two wind speed prediction models univariate model developed by ARIMA and the multivariate model developed by NARX. NARX presented accurate results. 
Zina \& Octavian [15] did the study on Prediction of daily direct solar radiation using NARX modeland they concluded that the model shows powerful results in the periodical training phase. M. A. Hamdan \& E. Abdelhafez [16] performed the prediction of hourly solar radiation in Amman with three types of neurons network: NARX, Feedforward and Elman. For their study case too, NARX was better and showed the best results in training and validation period of solar data prediction compared to other networks.

To evaluate the solar radiation of the Mutah city Yazeed \& Khaled [17] have built seven different models of NARX network by modifying input parameters, the number of neurons and the number of Time Delay. The results showed that the model has three parameters: temperature, humidity and wind speed is the most adequate to predict the solar radiation.

Lubna \& Mohammed [18] also used the NARX model for hourly solar radiation in Amman. To evaluate the NARX modeland examine his performance, they have kept the same model structure by modifying the training algorithms as: LM (Levenberg-Marquardt), RP (ResilientBackpropagation), SCG (Scaled Conjugate Gradient), CGP (Conjugate Gradient with Polak-Ribiére updates), CGF (Conjugate Gradientwith Fletcher-Reeves updates), CGB (Conjugate Gradient with Powell-Beale restarts) and OSS (One-Step secant backpropagation). The better training algorithm was LM.

There are other types of networks by which we can forecast the wind speed like CNN (Convolutional Neural Network). There are also others learning algorithms as SVR [19], SVM [20], Fuzzy Logics [21], ANFIS [22], Genetic algorithms [23].

\subsection{Input Variables}

Pretreatment of meteorological data was carried out in to facilitate modeling on NNTOOL (Neural Network Tool). To optimize the input number of our neural network and avoid the expensive calculation created by all the variables listed below we calculated the correlation coefficients between the input variables.

In Table 2, we observe that there is a strong correlation between temperature and dew point temperature, with the highest correlation coefficient 0,676 close to 1 that means that the dew point temperature has the same characteristics as temperature and it won't have influence on the training. This is the reason that allows us to neglect dew point temperature variable.

Table 2. Presentation of Correlation Coefficients between Variables

\begin{tabular}{cccccc}
\hline Variables & Wind Speed & Pressure & Temperature & Humidity & Dew point temperature \\
\hline Wind Speed & 1 & $-0,013959129$ & $-0,358440396$ & 0,163211507 & $-0,209401453$ \\
Pressure & & 1 & $-0,342550436$ & $-0,156650985$ & $-0,488960215$ \\
Temperature & & & 1 & $-0,19689524$ & 0,676457342 \\
Humidity & & & & 1 & 0,392057985 \\
Dew point temperature & & & & & 1 \\
\hline
\end{tabular}

\section{RESULTS AND DISCUSSION}

The neural network has been established with two different type of neuron networks (MLP) and (RNN) with two different algorithms, Levenberg-Marquardt back-propagation and Scaled Conjugate Gradient (SCG) successively [24].

After several try we changed every-time the number of inputs, from 3 input; Wind speed at (t-1), Pressure at (t-1) and Temperature at (t-1); to 4 inputs; Wind speed at (t-1), Pressure at (t-1), Temperature at $(\mathrm{t}-1)$ and humidity at (t-1); we changed also the numbers of neurons of hidden layer between 5 neurons and 25 neurons we found that the network who has 4 inputs; WS at (t-1), ALTM at (t-1) and TMP at (t-1) and H at (t-1); with 20 neurons on hidden layer present the better performances.

The error MSE (Mean Square Error) and RMSE (Root Mean Square Error) show the difference between the desired output and calculated output. They have been applied for all the cases mentioned before (from 3 input to 4 inputs, from 5 neurons to 25 neurons). The Table 3 present the best case found according to the tests, and since, we can conclude that a neural network with 4 inputs and 25 neurons in hidden layer presents the best case. We notice in Figure 5 that the values of $\mathrm{R}$ in the three training phase, Training, Validation and Test are $0.4021,0.45163$ and 0.52008 successively are very weak. This shows that the model is unable to predict correctly and achieve to performing results. Generally, we found that the LM backpropagation method gives weak results with $\mathrm{R}=0.4246$. 
Table 3. Table Allows to Find the Best Network's Architecture

\begin{tabular}{ccccc}
\hline & \multicolumn{2}{c}{3 Inputs } & \multicolumn{2}{c}{ 4 Inputs } \\
\hline & MSE & RMSE & MSE & RMSE \\
5 Neurons & 2,22933195 & 1,49309476 & 2,25208979 & 1,50069643 \\
10 Neurons & 2,24514902 & 1,49838213 & 2,25630653 & 1,50210071 \\
15 Neurons & 2,2338491 & 1,49460667 & 2,27750312 & 1,50913986 \\
20 Neurons & 2,21847102 & 1,48945326 & 2,19318051 & 1,48093906 \\
25 Neurons & 2,20455428 & 1,48477415 & 2,23454556 & 1,49483964 \\
\hline
\end{tabular}

With

MSE $=\frac{1}{n} \sum_{1}^{n}(O-d)^{2}$
RMSE $=\sqrt{\frac{1}{n} \sum_{1}^{n}(O-d)^{2}}$
O: Calculated Output
d: Desired Output

There are others statistical error models to analysis different algorithms as:

a) $\mathrm{R}^{2}$ : Coefficient de determination.

b) MBE: Mean Bias Error.

To optimize the forecast, improve performance, and minimize the error by changing the weights, we evaluated the networks (MLP) and (RNN) by two different algorithms:

a) Levenberg-Marquardt back-propagation: based on Gradient Back-propagation.

b) ScaledConjugate Gradient algorithm (SCG): based on Conjugate Gradient.

c) 1st case: Levenberg-Marquardt back-propagation

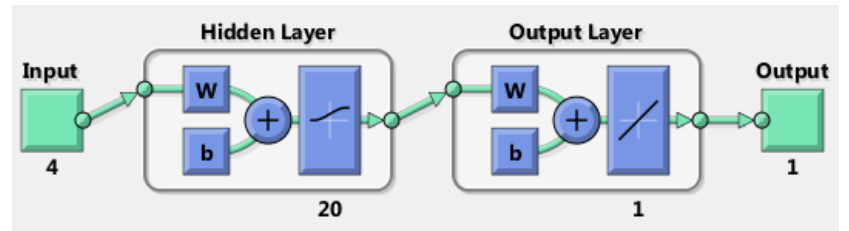

Figure 5. Presentation of MLP with back Propagation Levenberg-Marquardt Algorithm

The figures below present the training steps of the MLP network. Firstly, Training is used to fit the model it means that the model must sees and learns the data. Secondly, Validation is used to apply an evaluation of the fit of the model when setting parameters. The goal is to adjust the parameters (weight and bias) to find a powerful model. Finally, Test step that makes the last evaluation on the model so that it is more organized [25].
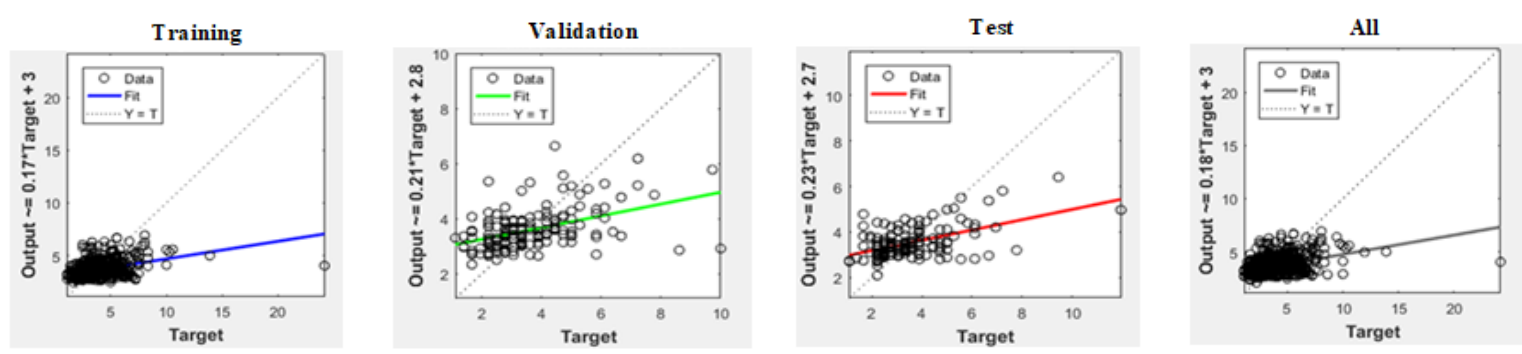

Figure 5. Regression rate presentation for levenberg-marquardt back propagation

Figure 6 shows the big difference between the prediction of the wind speed by the model and the real wind speed. We can see very well the difference between the two series. 2nd case: Scaled Conjugate Gradient (SCG). Presentation of NARX with Scaled Conjugate Gradient algorithm (SCG) as shown in Figure 7. 


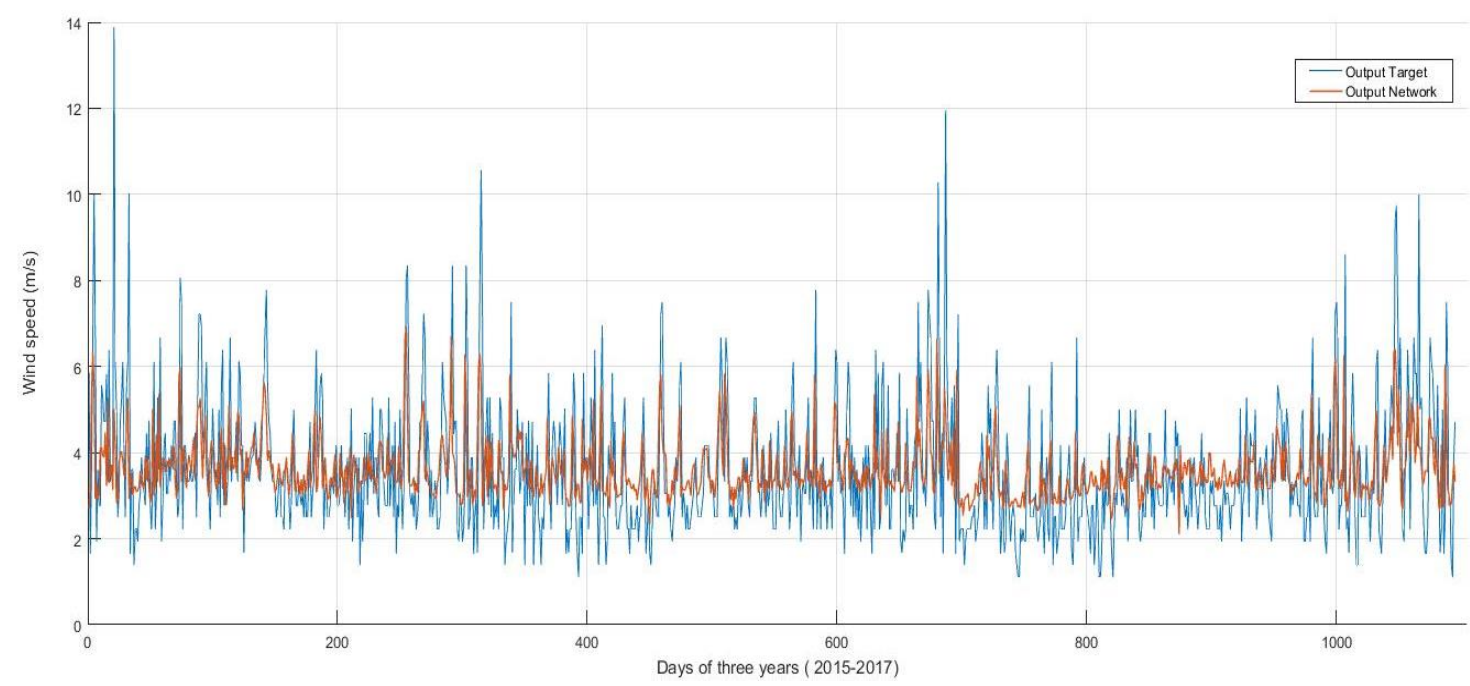

Figure 6. Wind speed prediction graph with Levenberg-Marquardt back-propagation model

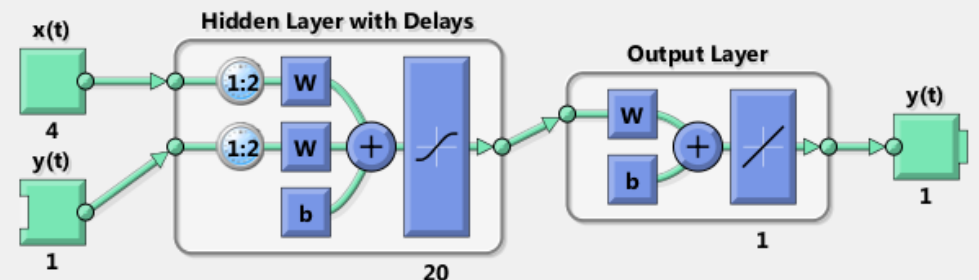

Figure 7. Presentation of NARX with Scaled Conjugate Gradient algorithm (SCG)

Our NARX model has 4 exogenous inputs in input layer, 20 neurons and hyperbolic tangent function with 2 delays time in hidden layer, and 1 endogenous output with linear function in output layer. Figure 8 presents the performance of the NARX model treated by Scaled Conjugate Gradient. Whose R is presented by $0.98454,0.98408$ and 0.98598 in training, validation and test period respectively. In comparison with the LM model, NARX data are identical to real wind speed data with $\mathrm{R}=0.98454$. The presentation of the two series; predict wind speed and real wind speed; the Figure 9 blow confirms that the NARX model shows a high performance in wind speed prediction.

In Table 4 we chose RMSE ( Root Mean Square Error ) because it's usually applied in climatology, forecast and resgression analysis cases to verify results in order to demonstrate that NARX model is more effective than the MLP model for our study case.
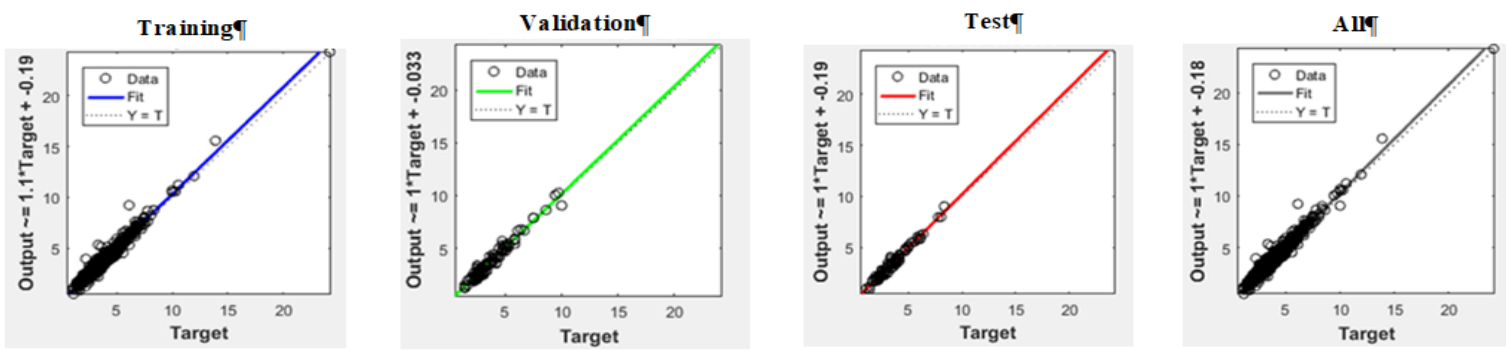

Figure 8. Regression rate presentation for scaled conjugate gradient 


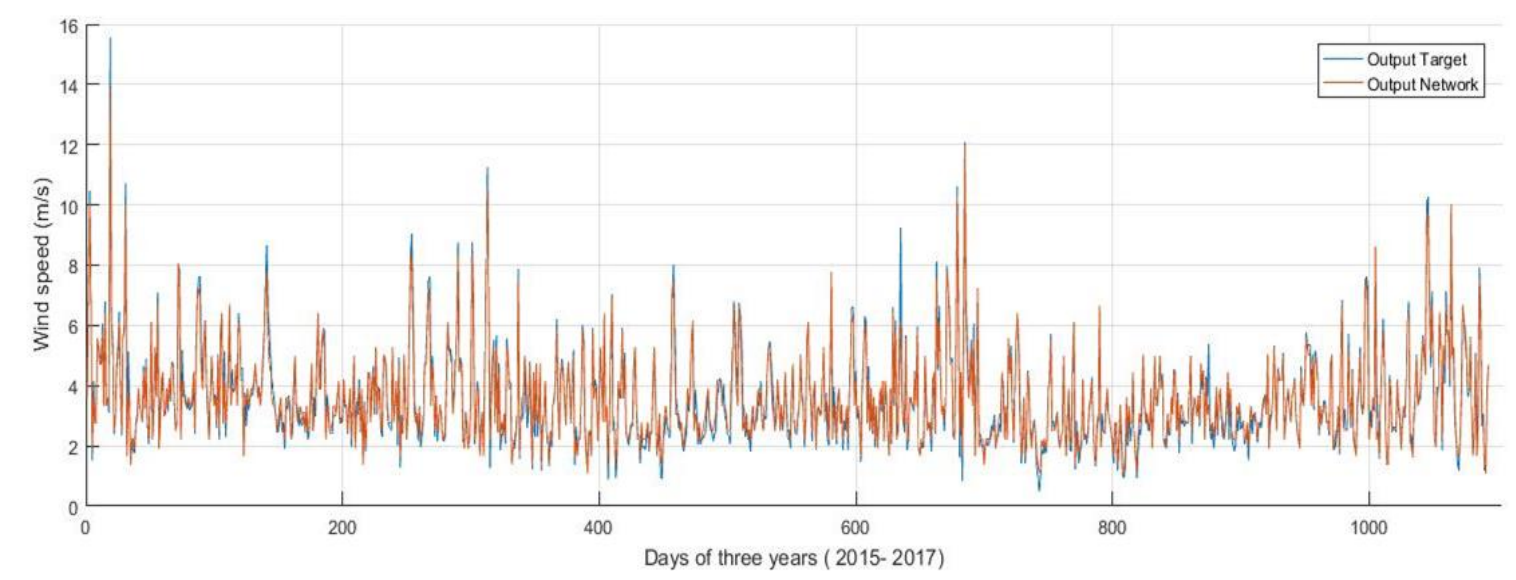

Figure 9. Wind speed prediction graph with NARX model

Table 4. Statistical Errors Generatered by the MLP Ans NARX Models

\begin{tabular}{cc}
\hline Model & RMSE \\
\hline MLP & 1,4809306 \\
NARX & 0,31455621 \\
\hline
\end{tabular}

\section{CONCLUSION}

Wind speed daily forecast of Tetouan region, has been done by two artificial neural networks, multilayer perceptron model (MLP) and recurrent neural network (RNN), which they keep the same structure defined by four inputs variables define the input layer $\mathrm{V}(\mathrm{t}-1), \mathrm{P}(\mathrm{t}-1), \mathrm{T}(\mathrm{t}-1)$ and $\mathrm{H}(\mathrm{T}-1)$, a hidden layer characterized by 20 neurons and an activation function. Wind speed at the instant $(t)$ defines the output layer. We used two different algorithms, Levenberg-Marquardt Back-propagation that it gave low results $\mathrm{R}=0,4246$, and model NARX trained by Scaled Conjugate Gradient. This last gave good results with $\mathrm{R}=0.98454$. Therefore, we can be concluded that NARX effectively treats nonlinear and complex problems better than MLPs.

\section{REFERENCES}

[1] R. Baile. "Analyse et modélisation multifractales de vitesses de vent. Application à la prévision de la ressource éolienne. Océan, Atmosphère," Université Pascal Paoli, (2010).

[2] M. Lei, et al., "A review on the forecasting of wind speed and generated power," Renewable and Sustainable Energy Reviews 13, 915-920 (2009).

[3] Y. Wang, et al., "Review on Studies and Advances of Machin Learning Approaches," TELKOMNIKA (Indonesian Journal of Electrical Engineering). 2014; 12(2): 1487-1494.

[4] X. Wang, et al., "A review of Wind Power Forecasting Models," Energy Procedia 12, 770-778 (2011).

[5] Costa, et al., "A review on the young history of the wind power short-term prediction," Renewable and Sustainable Energy Reviews 12, 1725-1744 (2008).

[6] G. Zhang, et al., "Forecasting with artificial neural networks: The state of art," International Journal of Forecasting 14, 35-62 (1998).

[7] A. Salami, et al., "Prédiction de la moyenne horaire de la vitesse du vent sur le site de Lomé par réseau de neurones," Vol. 2(1), ISSN 2312-8712 June (2017).

[8] K. Sreelakshmi, et al., "Neural Networks for Short Term Wind Speed Prediction," World Academy of Science, Engineering and Technology International Journal of Computer and Information Engineering. Vol:2, No:6 (2008).

[9] M. Bilgili, et al., "Application of artificial neural networks for the wind speed prediction of target station using reference stations data," Renewable Energy, 32, 2350-2360 (2007).

[10] M. W. Gardner, et al., "Artificial Neural Networks (The multilayer perceptron)-A review of Application in the atmospheric sciences," School of Environmental Sciences, University of East Anglia, Norwich, Norfolk NR4 7TJ, UK (1998).

[11] S. Benkachcha, et al., "Seasonal Time Series Forecasting Models based on Artificial Neural Network," International Journal of Computer Applications, (0975-8887) Volume 116-No. 20 April (2015).

[12] M. Yuhendri, et al., "Maximum Output Power Tracking of Wind Turbine Using Intelligent Control," TELKOMNIKA (Indonesian Journal of Electrical Engineering). 2011; 9(2): 217-226. 
[13] Q. Lyu, et al., "Revisit Long Short-Term Memory: An Optimization Perspective," Workshop on Deep Learning and Representation Learning (2014).

[14] E. Cadenas, et al., "Wind Speed Prediction Using a Univariate ARIMA Model and a Multivariate NARX Model," Energies, Volume 9, Issue 2 (2016).

[15] Z. Boussaada, et al., "A Nonlinear Autoregressive Exogenous (NARX) Neural Network Model for the Prediction of the Daily Direct Solar Radiation," Energies, Volume 11, Issue 3 (2018).

[16] M. A. Hamdan, et al., "Prediction of Hourly Solar Radiation in Amman-Jordan by Using Artificial Neural networks," Int.J.of Thermal \& Environmental Engineering, Volume 14, No.2 103-108 (2017).

[17] Y. A. Al Sbou, et al., "Nonlinear Autoregressive Recurrent Neural Network Model For Solar Radiation Prediction," International Journal of Applied Engineering Research, ISSN 0973-4562 Volume 12, Number 14 pp. 4518-4527 (2017).

[18] L. B. Mohammed, et al., "Hourly Solar Radiation Prediction Based on Nonlinear Autoregressive Exogenous (NARX) Neural Network, "Jordan Journal of Mechanical and Industrial Engineering. Volume, Number 1, December (2013).

[19] H. Wang, et al., "Electricity Consumption Prediction based on SVR with Ant Colony Optimization. TELKOMNIKA (Indonesian Journal of Electrical Engineering)," 2013; 11(11) : 6928-6934.

[20] J. Wang, et al., "A robust combination approach for short-term wind speed forecasting and analysis-Combination of the ARIMA (Autoregressive Integrated Moving Average), ELM (Extreme Learning Machine), SVM (Support Vector Machine) and LSSVM (Least Square SVM) forecasts using a GPR (Gaussian Process Regression) model," Energy 93 (2015).

[21] B. Sivaneasan, et al., "Solar Forecasting using ANN with Fuzzy Logic Pre-processing," World Engineers SummitApplied Energy Symposium \& Forum: Low Carbon Cities \& Urban Energy Joint Conference, WES-CUE 2017, 19-21 July 2017, Singapore. Energy Procedia 143,727-732 (2017).

[22] S. Jovic, et al., "Management of the wind speed data using adaptive neuro-fuzzy methodology," Flow Measurement and instrumentation, 002-07 (2016).

[23] H. Liu, et al., "New wind speed forecasting approaches using fast ensemble empirical model decomposition, genetic algorithm, Mind Evolutionary Algorithm and Artificial Neural Networks," Renewable Energy 83, 10661075 (2015).

[24] S. Nayak, et al., "Scaled Conjugate Gradient Backpropagation Algorithm for Selection of Industrial Robots," International Journal of Computer Application (2250-1797). Volume 7- No.6, November-December (2017).

[25] M. Caner, et al., "Investigation on thermal performance calculation of two type solar air collectors using artificial neural network," Expert Systems with Applications 28, 1668-1674 (2011). 